\title{
AN EXPONENTIAL AND LOGARITHMIC UTILITY APPROACH TO EIGENVECTOR METHOD IN THE ANALYTIC HIERARCHY PROCESS: THE IDEA VALIDATION WITH EXAMPLE LOGISTIC APPLICATION
}

\author{
Pawel Tadeusz Kazibudzki \\ Jan Dlugosz University in Czestochowa, Poland \\ E-mail: emailpoczta@gmail.com
}

\begin{abstract}
Thorough inventory control, planning and coordination, wise and thoughtful selection of warehousing and means of transport are necessary for efficient decisions making in logistics. In order to make optimal decisions, it is necessary to deliberate many options from the perspective of different criteria. It was empirically verified that such problems are difficult to solve without methodological support as they exceed humans' perception capabilities. However, there are some techniques which can facilitate the process of decision making when successfully applied. One of the most commonly used technique for structured problems solving is the Analytic Hierarchy Process. It is popular because its prescribed procedure supports sophisticated mathematical theory called the Eigenvalue Method which is accurate and unique. It does not mean that there are not other methods that can support the Analytic Hierarchy Process although their application has pros and cons. Two relatively novel methods were proposed recently and their validation studies are the essential part of this research. In comparison with others, their simplicity and compliance with the Eigenvalue Method are their most crucial but not exclusive advantages. At the end of the research, the example logistic problem was solved with their application, and the results of all methods were compared.
\end{abstract}

Keywords: Analytic Hierarchy Process, eigenvalue method, optimization models, decision support techniques, selection of transportation modes.

\section{Introduction}

Obviously problem solving activities pervade many aspects of human life and its levels. Additionally, due to the interdependent nature of most problems, they can also influence and be affected by other problems what constantly takes place in the real life at different levels of human activity. Generally, problem solving process is considered to be a multi-stage process and although many models and frameworks have been proposed in a literature, one of the most popular is Herbert Simon's (1960) categorization assuming its three phases: intelligence, design, and choice. The intelligence phase concerns the problem identification. In this stage the problem is recognized and information are gathered in order to formulate a problem definition. Design phase refers to the identification of alternative solutions to the problem. In the choice phase, the solution alternatives are selected and implemented. From the three different phases of problem solving mentioned above, the act of decision making (i.e. choice) has received the most attention in the academic literature. It is so, probably because we as the human beings, are unable to analyze simultaneously many different competing factors and then synthesize them 
PROBLEMS

OF MANAGEMENT

IN THE $21^{\text {st }}$ CENTURY

volume 2, 2011

for the purpose of rational decision. There is overwhelming scientific evidence showing that the unaided human brain is simply not capable to do it. That is why one needs some decision support tools which can provide him/her with the required assistance. Psychologists have proven that the human brain is limited in both its short term memory capacity and its discrimination ability. It was scientifically verified that a human being will give inaccurate answers when forced to choose from a range of twenty alternatives, because the range exceeds man's bandwidth of perception channel (Martin, 1973). It has been demonstrated that humans are not capable of dealing accurately with more than about seven things at a time. It is crucial to notice that results of numerous psychological experiments, including the well known Miller's study (Miller, 1956) verified this notion.

\section{Example Techniques for Decision Making Facilitation}

\section{Advantage-Disadvantage Procedure to Decision Analysis}

This technique is perhaps the most simple and fundamental of all the evaluation methods. On the other hand, it is also the approach that probably is used most frequently what does not necessarily mean that it is used effectively. The process typically uses a list of all alternatives, which are then separately examined from the perspective of their strengths and weaknesses against certain criteria. Finally, it is chosen the one that best fits the problem objective. The choice is made either on the bases of greatest number of advantages or largest net number of pros over cons (Van Gundy, 1981). Obviously, the primary limitation of the technique lies in the assumption that all criteria must have the same weights what seems very unlikely and constitutes the significant simplification of the whole problem.

\section{Weighting System Approach for Decision Making Support}

In order to avoid the main drawback of the previous method, this commonly used procedure involves assigning weights to the different evaluation criteria. Since not all criteria are probably assigned the same weights, the weighting system approach provides a systematic method for assessing the strengths and weaknesses of each alternative. The general steps this method entails constitute: creating an evaluation criteria list, assigning weights to the criteria, rating each alternative against the criteria and selecting the alternative that best satisfies the criteria on the bases of total points (Van Gundy, 1981). Obviously, at the end of the process the question arises, whether the attempt undertaken in order to quantify value preferences for both criteria and alternatives was valid. Let us remember that any measurement system is only as useful as the criteria applied to develop it and the information credibility, the ratings were based on. Moreover, there are two basic kinds of judgments: absolute and relative. However, it seems that humans can make much better relative judgments than absolute ones. It is so because they have the better ability to discriminate between the members of a pair than compare one thing against some recollection from their long term memory (Saaty, 2000).

\section{The Analytic Hierarchy Process}

It seems that making pairwise comparisons is for human being as natural as binary counting is for computers. It is so because humans have limited, so called bandwidth of their channel for perception. As it was indicated (Martin, 1973, p. 334), we can deal simultaneously only with about seven units of information, and this is our optimal performance as far as complex problem solving is concerned. Fortunately, there are some ways to improve our efficiency. 
The first is to enable a man making rather relative than absolute judgments. The second is to organize tasks into groups in order to make several judgments in succession. All of this is just the reason why the Analytic Hierarchy Process (AHP) uses pairwise comparisons. The method was developed at the Wharton School of Business by Thomas Saaty (1980) and since its creation has been applied to numerous decisional problems (Saaty, 2001). It has substantial theoretical and empirical support including the studies of human judgmental processes by cognitive psychologists. It overcomes the weaknesses of advantage-disadvantage procedure to decision analysis and the weighting system technique by using a hierarchical structure of the decision problem, pairwise relative comparison of the elements in the hierarchy, and a series of independent judgments. The AHP method reduces in this way possible inaccuracy of human's judgments and provides a measure of their consistency. The first stage in AHP model building is to decompose the overall problem into a hierarchy consisting, minimally, of a goal, criteria, and alternatives. Each element, or level, in the hierarchy can be delineated further into another set of manageable components. For example, criteria can be decomposed into subcriteria. The hierarchical structuring continues down to the most specific component of the problem, which usually involve specific alternatives or courses of action available to the decision maker. There are many possible hierarchical models that can be applied to a wide variety of problems. The following ones may be included as some of them (Dyer et al., 1991, p. 89):

- goal, criteria, alternatives;

- goal, criteria, subcriteria, alternatives;

- goal, criteria, subcriteria, scenarios, alternatives;

- goal, actors, criteria, alternatives;

- goal, criteria, levels of intensities, many alternatives;

The second stage in this process is to establish priorities among the elements at each level of the hierarchy. The decision maker evaluates (in a pairwise manner) the relative importance, preference, or likelihood of each set of elements with respect to elements at the immediately higher level in the hierarchy. First pairwise comparisons of the relative preference for the alternatives are made with respect to each of the lowest level (sub) criteria. Next, pairwise comparisons are made about the relative importance of the subcriteria with respect to each criterion, and then for the relative importance of the top level criteria with respect to the goal. The process of making pairwise comparisons uses nine-point scale in order to evaluate preferences for each pair of items. The scale provides both numerical and corresponding verbal expressions for each judgment and is commonly available in the literature (Saaty, 2001, p. 26). Finally, the last stage involves synthesising according to a well-prescribed procedure (standard AHP aggregation based on weighting and adding) and calculation of so called inconsistency ratio that reflects the degree of judgments inconsistency.

Recapitulating, that what makes AHP so different and effective from other similar methods is that (A) it enables making judgments in the verbal way, and (B) it entails mathematical analysis performed on the judgments which provides relatively very accurate results and the measure of their inconsistency.

However, it is the mathematical analysis i.e. the eigenvalue method, which the AHP applies, that despite of its popularity, has as well its share of criticism. For instance some authors have noticed that Saaty's procedure does not optimize any objective function, what entails it cannot be interpreted in statistical or optimization fashion. In particular, solutions obtained with the application of the eigenvalue method in the AHP cannot be compared to other ones received with an application of different commonly known methods (Choo et al., 2004; Crawford et al., 1985). Besides, unlike many optimization models, the method applied by the AHP does not allow a decision maker for introduction of additional constraints that might be seemed necessary according to his/her point of view (Lam et al., 1995). Moreover, the method 
PROBLEMS

OF MANAGEMENT

IN THE $21^{\text {st }}$ CENTURY

Volume 2, 2011

commonly applied to the AHP is supposed to operate only with, so called, reciprocal pairwise comparisons matrices what entails a limited range of application. Another drawback of the analyzed procedure was revealed quite recently (Bana e Costa, 2008), and proved that the eigenvalue method, commonly applied to the AHP, does not satisfy a so called condition of order preservation.

\section{The Eigenvector Method in the AHP}

In order to derive priorities from pairwise comparisons AHP uses a principal Eigenvalue Method (EM) which has been recently a subject of more detailed analysis (Kazibudzki, 2011a). Generally speaking a problem of deriving priority weights from so called pairwise comparison matrix (PCM) denoted as $\boldsymbol{A}=\left[a_{i j}\right]_{\mathrm{nnn}}$ with elements $a_{i j}=a_{i} / a_{j}$ is to estimate a priority vector (PV) $w=\left[w_{1}, w_{2}, w_{3}, \ldots, w_{n}\right]^{T}$ on the bases of the matrix $\boldsymbol{A}$ which comprises a decision maker (DM) pairwise comparisons judgments concerning the importance of a given binary set of alternatives. Commonly the priority weights $w_{i}, i=1, \ldots, n$, are chosen to be

positive and normalized to unity: $\sum^{n} w_{i}=1$, and the elements $a_{i j}$ of the matrix $\boldsymbol{A}$ are then the DM judgments about the priority $i \quad$ ratios $w_{i} / w_{j}, i, j=1, \ldots, n$, where $n$ is the number of all alternatives being considered. In a perfect judgment case then, one has:

$$
A \times w=n \times w[1]
$$

and in this situation the PV $\boldsymbol{w}$ can be computed by solving that so called eigenvector equation. It is so because the number $n$ in the perfect case (matrix $\boldsymbol{A}$ is consistent) is the principal eigenvalue of $\boldsymbol{A}$, i.e. the largest solution of the characteristic equation:

$$
\operatorname{det}(\boldsymbol{A}-\mathrm{lambda} \times \mathbf{I})=0 \text { [2] }
$$

where $I$ denotes identity matrix of order $n$. In this case it is also the only nonzero eigenvalue. On the other hand, when the case is not perfect (matrix $\boldsymbol{A}$ is inconsistent) an estimate of the true PV is normalized right eigenvector associated with the largest eigenvalue. Thus, in order to obtain the estimate one needs to solve general eigenvector equation:

$$
\boldsymbol{A} \times \mathbf{w}=l a m b d a_{\text {max }} \times \boldsymbol{w} \text { [3] }
$$

where $l a m b d a_{\text {max }}$ denotes the principal eigenvalue which is not smaller than $n$, is simple and its existence is guaranteed by Perron's Theorem (Saaty, 1994), while other lambdas are close to zero.

If the elements of a matrix $\boldsymbol{A}$ satisfy the condition $a_{i j}=1 / a_{j i}$ for all $i, j=1 \ldots n$ then the matrix $\boldsymbol{A}$ is said to be reciprocal (RPCM). If its elements satisfy the condition $a_{i k} a_{k j}=a_{i j}$ for all $i$, $j, k=1 \ldots n$ and the matrix is reciprocal, then it is called consistent. Finally, the matrix $\boldsymbol{A}$ is said to be transitive if the following conditions hold: (A) if for any $l=1, \ldots, n$, an element $a_{l j}$ is not less than an element $a_{l k}$ then $a_{i j} \geq a_{k}$ for $i=1, \ldots, \mathrm{n}$, and (B) if for any $l=1, \ldots, n$, an element $a_{j l}$ is not less than an element $\quad a_{k l}$ then $a_{j i} \geq a_{k}$ for $i=1, \ldots, n$. Obviously, in the case of the reciprocal PCM the two conditions (A) and (B) are equivalent.

\section{An Exponential and Logarithmic Utility Approach to EM in the AHP}

It has been already deduced (Grzybowski, 2010) that instead of solving the eigenvalue equation [3] one may seek a vector $\boldsymbol{w}$ which best estimates the equation [1]. So, in order to satisfy the equation [1] as perfectly as possible it has been recently proposed (Kazibudzki, 2011a) to estimate the PV by solving one of the following constrained optimization problems: 


$$
\begin{aligned}
& \text { PROBLEMS } \\
& \min \sum_{i=1}^{n}\left(\exp \left(\sum_{j=1}^{n} a_{i j} w_{j}-w_{i}\right)-1\right)^{2}
\end{aligned}
$$

subject to:

$$
\sum_{i=1}^{n} w_{i}=1, \quad w_{i} \geq 0, \quad i, j=1, \ldots, n
$$

or respectively (Kazibudzki, 2011b):

$$
\begin{aligned}
\min \sum_{i=1}^{n}\left(\mathrm{~h}\left(\sum_{j=1}^{n} \frac{a_{i j} w_{j}}{w_{i}}\right)\right)^{2} \text { subject to: } & \\
& \sum_{i=1}^{n} w_{i}=1, \quad w_{i}>0, \quad i, j=1, \ldots, n
\end{aligned}
$$

Because the utility of both methods decreases exponentially or logarithmically, inversely proportionally to the inconsistency ascend, it seems natural that they can be commonly identified, respectively as the Exponential Utility Approach (EUA) and the Logarithmic Utility Approach (LUA) to the problem of priorities weighting. The basic concept of the above two methods is to minimize a distance between an ideal unknown PV giving consistent PCM and a PV resulting from a DM judgments, typically involving inconsistent PCM. The quest for this solution with the application of commonly known constrained optimization procedures results with the PV which best fits to the ideal one delivering consistent PCM. In the next section EM will be compared to the two approaches with the application of computer simulations.

The Validation Studies of EUA and LUA to EM in the AHP

In the simulations with the help of sophisticated mathematical software the following types of random inconsistent PCMs were generated: reciprocal and transitive (RTPCM), only reciprocal (RPCM), and only transitive (TPCM). In order to compare results obtained from EUA and LUA with results given by EM, firstly ten thousands of RTPCMs were generated, in order to find out if there is any possibility of rank reversal phenomena between the methods, and secondly one thousand matrices from each of remaining types (RPCM and TPCM). For every matrix the PVs: $\boldsymbol{w}_{\mathrm{EUA}}, \boldsymbol{w}_{\mathrm{LUA}}$, and $\boldsymbol{w}_{\mathrm{EM}}$ were calculated with the application of EUA, LUA and EM, respectively. Next were computed: Pearson correlation coefficient (PCC) between the priority vectors and Spearman rank correlation coefficient (SRCC) between the priority ranks. The considered number of decision alternatives $n$ was: 3, 5, 7, 9, 11 in the case of RTPCMs and, for variety reasons: 4, 6, 8, 10 in the case of remaining types of PCMs, i.e. RPCM and TPCM. For the purpose of preference ratios evaluations, which entail the scope of elements in PCMs, the standard AHP scale was applied, i.e. integers: $1, \ldots, 9$ and their inverses. Table 1, 2, and 3 show the mean correlation coefficients between priority vectors and priority ranks generated 
PROBLEMS OF MANAGEMENT IN THE $21^{\text {st }}$ CENTURY Volume 2, 2011

with the application of the two novel methods (EUA, LUA) and EM, on the bases of the whole set of data in relation to a given type of PCM and number of alternatives.

Table 1. Simulation results of comparative studies EUA and LUA versus EM for RTPCMs.

\begin{tabular}{|c|c|c|c|c|}
\hline \multirow{2}{*}{$\begin{array}{c}\text { Number of } \\
\text { alternatives " } n \text { " }\end{array}$} & \multicolumn{4}{|c|}{ RTPCM } \\
\cline { 2 - 5 } & PCC & SRCC & PCC & SRCC \\
\cline { 2 - 5 } & 1 & 1 & 0,999965 & 0,9999116 \\
\hline $\mathrm{n}=3$ & 0,999997 & 1 & 0,999891 & 0,999742 \\
\hline $\mathrm{n}=5$ & 0,999991 & 1 & 0,999874 & 0,999841 \\
\hline $\mathrm{n}=7$ & 0,999991 & 1 & 0,999906 & 0,999924 \\
\hline $\mathrm{n}=9$ & 0,999997 & 1 & 0,999887 & 0,999876 \\
\hline $\mathrm{n}=11$ & & & & \\
\hline
\end{tabular}

Table 2. Simulation results of comparative studies EUA and LUA versus EM for RPCMs.

\begin{tabular}{|c|c|c|c|c|}
\hline \multirow{2}{*}{$\begin{array}{c}\text { Number of } \\
\text { alternatives " } \mathrm{n} \text { " }\end{array}$} & \multicolumn{4}{|c|}{ RTPCM } \\
\cline { 2 - 5 } & PCC & SRCC & PCC & SRCC \\
\cline { 2 - 5 } & 0,996699 & 0,995 & 0,993527 & 0,974122 \\
\hline $\mathrm{n}=4$ & 0,980279 & 0,956914 & 0,950362 & 0,905241 \\
\hline $\mathrm{n}=6$ & 0,961772 & 0,929262 & 0,873306 & 0,741204 \\
\hline $\mathrm{n}=8$ & 0,944564 & 0,930303 & 0,796861 & 0,630861 \\
\hline $\mathrm{n}=10$ & &
\end{tabular}

Table 3. Simulation results of comparative studies EUA and LUA versus EM for TPCMs.

\begin{tabular}{|c|c|c|c|c|}
\hline \multirow{2}{*}{$\begin{array}{c}\text { Number of } \\
\text { alternatives " } n \text { " }\end{array}$} & \multicolumn{4}{|c|}{ RTPCM } \\
\cline { 2 - 5 } & PCC & SRCC & PCC & SRCC \\
\cline { 2 - 5 } & 0,998773 & 0,998779 & 0,99882 & 0,997528 \\
\hline $\mathrm{n}=4$ & 0,998479 & 0,998992 & 0,999181 & 0,997989 \\
\hline $\mathrm{n}=6$ & 0,998619 & 0,998911 & 0,999238 & 0,99563 \\
\hline $\mathrm{n}=8$ & 0,998844 & 0,999044 & 0,99934 & 0,997083 \\
\hline $\mathrm{n}=10$ & &
\end{tabular}

As it can be seen from tables 1, 2 and 3 the validation studies of EUA and LUA versus EM can be successfully perceived. The very essential result of them is the score of the RTPCMs simulations for LUA which prove that this method fully complies with EM giving the same rank order in all ten thousands cases of simulated matrices $(\mathrm{SRCC}=1)$ and for every studied set of alternatives $(n=3,5,7,9,11)$. Amazingly close relation between studied methods can be also found in the case of RPCMs and TPCMs, however let us remember that in this case one has to be conscious that EM is designed only for the application with reciprocal matrices and because of that in the case of matrices which are nonreciprocal the EUA and LUA have their advantage over EM, what may also certify that they have much broaden range of applications and can be adopted in cases where EM simply fails. Together with other important findings concerning 
EUA (Kazibudzki, 2011a) and LUA (Kazibudzki, 2011b) it can be concluded, that there is at least one valid method for deriving the priority vector from a pairwise comparison matrix,

PROBLEMS OF MANAGEMENT IN THE $21^{\text {st }}$ CENTURY Volume 2, 2011 particularly when the matrix is inconsistent, which is equally satisfying as eigenvalue method and sometimes it is even better. This method is LUA and it is so because it fully complies with EM, it can be applied to both reciprocal and nonreciprocal matrices, it is computationally simpler and what is most important it does not violate condition of order preservation (Kazibudzki, 2011b). Obviously, analyzed here approach needs further studies and analysis to be strengthen.

Further, both presented and analyzed above methods will be used for the purpose of logistics application.

\section{Selecting Transportation Modes - an Example of EUA and LUA Application}

It is a fact that physical distribution of goods can represent a major cost category for many companies. Due to poor planning, lack of coordination and lost or damage shipments additional costs must be unnecessarily incurred. However, it is possible to avoid this burden through thorough inventory control, planning and coordination, wise and thoughtful selection of warehousing and means of transport. Undoubtedly, during transportation modes selection, the cost constitutes the most important criterion for decision makers. However, there are also other criteria that can affect an overall customer service which are recklessly neglected in the selection process. In order to overcome this situation, it is proposed to apply in such cases the AHP model presented below which offers a means of reviewing alternatives from different perspectives on the bases of different criteria. It seems worth to notice, that slight adaptation of this model can also assist in the evaluation of the more general modes of transportation like for instance rail, air, water and trucking, including formerly selected specific carriers within each mode (Kotler, 1988, p. 747). The model is the simple one. Under the overall goal (the optimal mode of transportation selection), there are four criteria: c1 (delivery time), c2 (availability), c3 (cost of shipments), and c4 (conformance with package size and weight requirements). Under each criterion, there are four alternatives: a1 (carrier no.1), a2 (carrier no.2), a3 (carrier no.3), and a4 (carrier no.4), which are the same for all the four criteria. The example judgment matrices and corresponding estimation of PVs, as well as overall PV, obtained with the application of EM, LUA, and EUA, respectively, are provided below:

\begin{tabular}{|c|c|c|c|c|c|c|}
\hline c1 & c2 & c3 & c4 & EM & LUA & EUA \\
\hline$[1$ & 2 & 3 & 67 & {$[0.4637]$} & $0.4655]$ & 0.4761 \\
\hline $1 / 2$ & 1 & 3 & 3 & 0.2943 & 0.2935 & 0.2998 \\
\hline $1 / 3$ & $1 / 3$ & 1 & 5 & 0.1772 & 0.1764 & 0.1700 \\
\hline $1 / 6$ & $1 / 3$ & $1 / 5$ & 1 & 0.0648 & 0.0646 & 0.0541 \\
\hline
\end{tabular}

with respect to the GOAL:

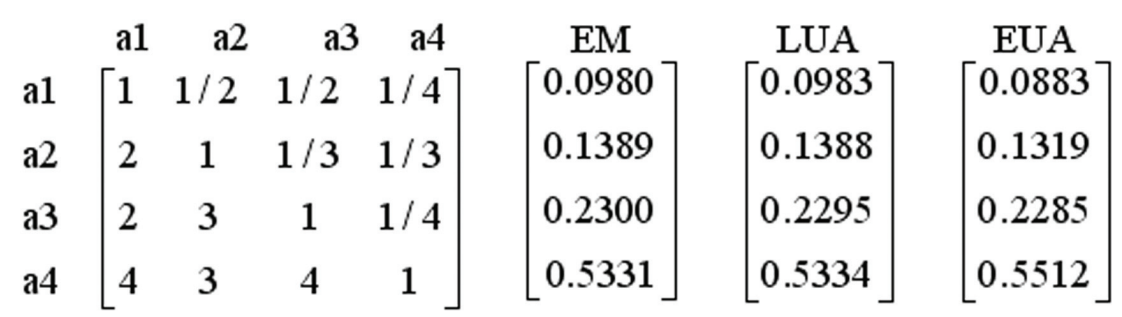


PROBLEMS OF MANAGEMENT IN THE 21 $1^{\text {st }}$ CENTURY
Volume 2, 2011

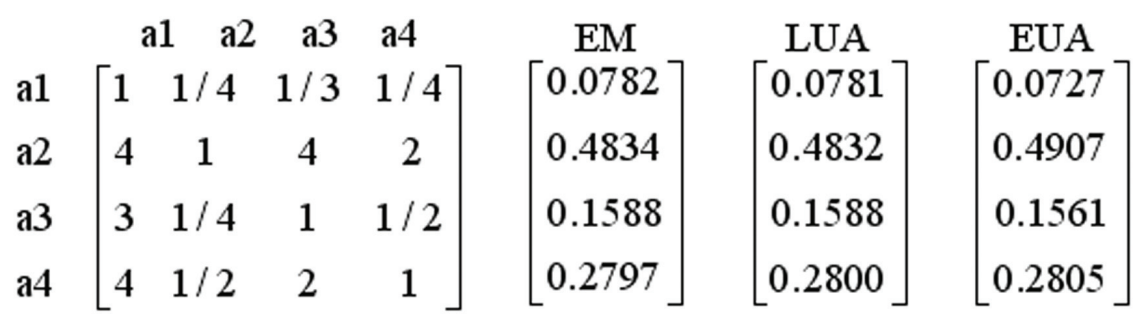

with respect to criterion $\mathrm{c} 2$ :

\begin{tabular}{|c|c|c|c|c|c|c|}
\hline a1 & a2 & a & a4 & $\mathrm{EM}$ & LUA & EUA \\
\hline$\lceil 1$ & $1 / 4$ & $1 / 3$ & $1 / 4$ & 0.0680 & 0.0717 & 0.0265 \\
\hline 4 & 1 & 4 & $1 / 2$ & 0.3778 & 0.3759 & 0.4024 \\
\hline 3 & $1 / 4$ & 1 & 2 & 0.2456 & 0.2444 & 0.2479 \\
\hline 4 & 2 & $1 / 2$ & 1 & 0.3086 & 0.3081 & 0.3232 \\
\hline
\end{tabular}

with respect to criterion $\mathrm{c} 3$ :

\begin{tabular}{|c|c|c|c|c|c|c|}
\hline a1 & a2 & $a^{3}$ & a4 & $\mathrm{EM}$ & LUA & EUA \\
\hline$[1$ & 2 & 1 & $1 / 3]$ & {$[0.1797$} & 0.1798 & 0.1790 \\
\hline $1 / 2$ & 1 & $1 / 5$ & $1 / 5$ & 0.0945 & 0.0945 & 0.0935 \\
\hline 1 & 5 & 1 & $1 / 4$ & 0.1682 & 0.1682 & 0.1677 \\
\hline 3 & 5 & 4 & 1 & 0.5576 & 0.5576 & 0.5599 \\
\hline
\end{tabular}

with respect to criterion $\mathrm{c} 4$ :

After synthesis, the following overall ranking is obtained:

\begin{tabular}{|c|c|c|}
\hline EM & LUA & EUA \\
\hline$[0.0921$ & {$[0.0929$} & {$[0.0780$} \\
\hline 0.2797 & 0.2788 & 0.2834 \\
\hline 0.2078 & 0.2074 & 0.2068 \\
\hline 0.4203 & 0.4208 & 0.4317 \\
\hline
\end{tabular}

It is easy to notice that all three methods: the Eigenvalue Method, Logarithmic Utility Approach and Exponential Utility Approach, provide the same overall alternatives ranks, resulting: a4>a2>a3>a1. Obviously, it means that in this hypothetic example "carrier no. 4" should be the ultimate choice of a decision maker.

\section{Conclusions}

Thorough inventory control, planning and coordination, wise and thoughtful selection of warehousing and means of transport are necessary for efficient decisions making in logistics. In order to make optimal decisions, it is necessary to deliberate many options from the perspective of different criteria. It was empirically verified that such problems are difficult to solve without methodological support, as they exceed humans' perception capabilities. However, 
one has already got techniques which application can help. One of the most commonly used for structured problems solving is the Analytic Hierarchy Process. It is so popular because its prescribed procedure supports sophisticated mathematical theory called the Eigenvalue Method which is accurate and unique. However, two new and relatively novel methods were proposed recently. Their simplicity and compliance with the Eigenvalue Method are their most crucial but not exclusive advantages which proved the validation studies provided in this research. At the end of the paper, the example logistic problem was solved with their application, and the results of all methods were compared as well.

\section{Acknowledgements}

I would like to express my gratitude to Andrzej Z. Grzybowski for his support during the phase of article preparation, and especially for designing the sophisticated software, without which validation studies of the methods being the research subject of this paper, would not be possible.

\section{References}

Bana e Costa, C. A., Vansnick, J. C. (2008). A critical analysis of the eigenvalue method used to derive priorities in AHP. European Journal of Operational Research, Vol. 187, p. 1422-1428.

Choo, E. U., Wedley, W. C. (2004). A common framework for deriving preference values from pairwise comparison matrices. Computers \& Operations Research, Vol. 31, p. 893-908.

Crawford, G., Williams, C. A. (1985). A note of the analysis of subjective judgment matrices. Journal of Mathematical Psychology, Vol. 29, p. 387-405.

Dyer, R. F., Forman, E. H. (1991). An analytic approach to marketing decisions. Prentice Hall, New Jersey.

Grzybowski, A. Z. (2010). Goal programming approach for deriving priority vectors - some new ideas. Scientific Research of the Institute of Mathematics and Computer Science, Vol. 1(9), p. 17-27.

Kazibudzki, P. T. (2011a). An exponential utility approach to eigenvector method in the analytic hierarchy process: an idea intro. Problems of Management in the $21^{\text {st }}$ Century, Vol. 1, p. 60-66.

Kazibudzki, P. T. (2011b). A scenario based analysis of a logarithmic utility approach for deriving priority vectors in the analytic hierarchy process. Scientific Research of the Institute of Mathematics and Computer Science, [submitted to Journal].

Kotler, P. (1988). Marketing Management: Analysis, Planning, Implementation, and Control. $6^{\text {th }}$ Ed., Prentice Hall, Englewood Cliffs, New York.

Lam, K. F., Choo, E. U. (1995). Goal programming in preference decomposition. Journal of Operational Research Society, Vol. 46, p. 205-213.

Martin, J. (1973). Design of Man-Computer Dialogues. Prentice Hall, Englewood Cliffs, New York.

Miller, G. A. (1956). The magical number seven, plus or minus two: some limits on our capacity for information processing, Psychological Review, Vol. 63, p. 81-97.

Saaty, T. L. (2000). The Brain: Unraveling the Mystery of How it Works. RWS Publications, Pittsburgh, pp. 3-24.

Saaty, T. L. (2001). Decision Making for Leaders. The Analytical Hierarchy Process for Decisions in a Complex World. RWS Publications, Pittsburgh, pp. 93-251.

Saaty, T. L. (1980). The Analytic Hierarchy Process. Mcgraw Hill, New York. 
OF MANAGEMENT

IN THE $21^{\text {st }}$ CENTURY

Volume 2, 2011

Saaty, T. L. (2001). Decision Making with Dependence and Feedback. The Analytic Network Process. RWS Publications, Pittsburgh.

Saaty, T. L. (1994). Fundamentals of the Analytic Hierarchy Process. RWS Publications, Pittsburg.

Simon, H. A. (1960). The New Science of Management Decision. Harper \& Brothers, New York, pp. 40 43.

Van Gundy, A. B. (1981). Techniques of Structured Problem Solving. Litton Educational Publishing, Inc., New York, pp. 147-188

Advised by Andrzej Z. Grzybowski, Technical University of Czestochowa, Czestochowa, Poland

Received: July 10, 2011

Accepted: September 25, 2011

Pawel Tadeusz Kazibudzki Dr., M.B.A., Assistant Professor, The Faculty of Social Sciences, Jan Dlugosz University in Czestochowa, Waszyngtona 4/8, 42-200 Czestochowa, Poland.

E-mail: emailpoczta@gmail.com

Website: http://www.ajd.czest.pl/p993,Kazibudzki 